\title{
MODELO DE GERENCIAMENTO DE PROCESSOS DE NEGÓCIO INTEGRADO AO GERENCIAMENTO DE RISCOS PARA A MELHORIA DO PROCESSO PRODUTIVO
}

\section{BUSINESS PROCESS MANAGEMENT MODEL INTEGRATED WITH RISK MANAGEMENT TO IMPROVE THE PRODUCTION PROCESS}

Recebido em: 13 nov. 2019

Aprovado em: 24 ago. 2020

Versão do autor aceita publicada online: 24 ago. 2020

Publicado online: 19 maio 2021

Como citar esse artigo - American Psychological Association (APA):

Guimarães, P. M. da S., Braga, J. L., \& Zaidan, F. H. (2021, out./dez.). Modelo de gerenciamento de processos de negócio integrado ao gerenciamento de riscos para a melhoria do processo produtivo. Exacta. 19(4), 890-910.https://doi.org/10.5585/exactaep.2021.16023.

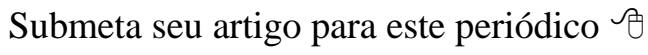

Dados Crossmark 


\title{
MODELO DE GERENCIAMENTO DE PROCESSOS DE NEGÓCIO INTEGRADO AO GERENCIAMENTO DE RISCOS PARA A MELHORIA DO PROCESSO PRODUTIVO
}

\author{
BUSINESS PROCESS MANAGEMENT MODEL INTEGRATED WITH RISK \\ MANAGEMENT TO IMPROVE THE PRODUCTION PROCESS
}

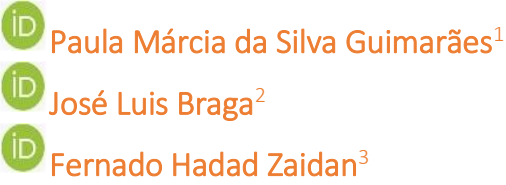

${ }^{1}$ Mestre

IETEC - Instituto de Educação Tecnológica, Mestrado em Engenharia e Gestão de Processos e Sistemas. Área de GRC-Governança, Risco e Compliance. Belo Horizonte, Minas Gerais - Brasil. paula.guimaraes75@yahoo.com.br

${ }^{2}$ Doutor

IETEC - Instituto de Educação Tecnológica, Mestrado em Engenharia e Gestão de Processos e Sistemas. Área de GRC-Governança, Risco e Compliance. Belo Horizonte, Minas Gerais - Brasil. zeluisbraga@gmail.com

\section{${ }^{3}$ Doutor}

IETEC - Instituto de Educação Tecnológica, Mestrado em Engenharia e Gestão de Processos e Sistemas. Área de GRC-Governança, Risco e Compliance. Belo Horizonte, Minas Gerais - Brasil. fhzaidan@gmail.com

Recebido em: 13 nov. 2019

Aprovado em: 24 ago. 2020
Resumo: O mercado está cada vez mais competitivo e, por isso, as organizações têm adotado técnicas para garantir a própria sobrevivência. O Gerenciamento de Processos de Negócio (Business Process Management - BPM) vem sendo adotado por oferecer uma melhora significativa no desempenho organizacional. Entretanto, os processos enfrentam riscos que podem causar impactos em seus objetivos. A integração entre as técnicas de gerenciamento de processos de negócios e de gerenciamento de risco é chamado de R-BPM. Apesar da obtenção de resultados satisfatórios com a utilização do R-BPM, a base científica nesta área é escassa, com integração incompleta entre as técnicas e ausência de uma ferramenta adequada de apoio. Este artigo tem como objetivo suprir esta lacuna através da elaboração de um modelo genérico de integração de todas as fases do ciclo de vida BPM com o gerenciamento de riscos e da utilização de ferramentas de apoio adequadas. A proposta foi testada em um processo real e os resultados mostraram que o método, além de permitir a integração completa entre as técnicas, é amplamente eficaz.

Palavras-chave: Gerenciamento de riscos. Gerenciamento de processos de negócios. Gerenciamento de processos de negócios consciente dos riscos.

Abstract: The market is increasingly competitive and, therefore, organizations have adopted techniques to ensure their own survival. Business Process Management (BPM) has been adopted because it offers a significant improvement in organizational performance. However, processes are facing risks that can impact their objectives. The integration between business process management and risk management techniques is called R-BPM. Despite obtaining satisfactory results with the use of R-BPM, the scientific base in this area is scarce, with incomplete integration between techniques and the absence of an adequate support tool. This article aims to fill this gap by developing a generic model for integrating all phases of the BPM life cycle with risk management and the use of appropriate support tools. The proposal was tested in a real process and the results showed that the method, in addition to allowing complete integration between the techniques, is largely effective.

Keywords: Risk management. Business process management. Risk-aware business process management. 
1 Introdução

Mercados cada vez mais complexos, globais, dependentes de tecnologia e altamente competitivos fazem com que a concorrência entre as organizações aumente rapidamente. Como consequência, os clientes exigem o cumprimento de seus requisitos específicos, ou seja, itens obrigatórios de fornecimento que visam buscar uma melhoria contínua em: prazos, preços, segurança e, principalmente, na qualidade dos produtos e/ou serviços oferecidos, forçando as organizações a melhorarem continuamente seus processos de negócios. Frente a essa conjuntura, as organizações precisam ser mais adaptáveis às mudanças.

Com a aplicação de técnicas e definições da abordagem de Gerenciamento de Processos de Negócio (do inglês, Business Process Management - BPM) é possível que uma organização torne seus processos mais eficazes, com maior precisão, mais rapidez, alto desempenho, melhor flexibilidade e alta qualidade (Lamine et al., 2020; Mahmood et al., 2018; Ferreira et al., 2016; Hofstede, A. (2011); Hammer, 2010). Entretanto, mesmo implantando o BPM de forma eficaz e obtendo resultados satisfatórios, as atividades dos processos das organizações estão expostas às incertezas. Eventos adversos e impactos associados são comumente conhecidos como riscos (Lamine et al., 2020; Ferreira et al., 2016). Assim, é necessário gerenciá-los em todas as fases do ciclo de vida BPM, pois os processos estão expostos continuamente aos riscos, devendo assim, ser identificados e tratados (Baldam, 2014).

Alguns estudos salientam que a fase de identificação de riscos contribui para uma melhor compreensão da relação entre os riscos e processos alinhados aos objetivos estratégicos das organizações (Rosemann et al., 2005; Dickstein et al., 2008). Para a obtenção de um resultado satisfatório na fase de identificação de riscos é necessário que haja um compartilhamento eficaz do conhecimento dos envolvidos nos processos de negócios analisados. Neste contexto, os resultados desta fase servem como base para o desempenho adequado das fases subsequentes do ciclo de vida BPM e para a tomada de decisões dos responsáveis dos processos (Lamine et al., 2020; Ferreira et al., 2016).

O gerenciamento dos riscos em processos de negócios é tão importante que a integração tem sido tratada pelos pesquisadores como Gerenciamento de Processos de Negócio Consciente dos Riscos (do inglês, Risk-Aware Business Process Management) (Lamine et al., 2020; Ferreira et al., 2016; Varela, 2016; Haggag, Khedr, \& Montasser, 2015; Suriadi et al., 2014; Hofstede, A. 2011).

Os objetivos do R-BPM propõem resultados robustos e satisfatórios em ambientes incertos (Lamine et al., 2020). Porém, o principal problema dos trabalhos existentes na literatura é que a integração do gerenciamento de riscos com todas as fases do ciclo de vida BPM é exposta de forma incompleta. Islam et al. (2009), Cope et al. (2010), Conforti et al. (2011) e Haggag, Khedr, \& Montasser 
(2015), Ferreira et al. (2016), por exemplo, não efetuam uma integração da abordagem de riscos com todas as fases do ciclo de vida BPM, sendo demonstrado na seção 2.4 a seguir.

Com a finalidade de preencher a lacuna existente na literatura, este artigo propõe uma aplicação prática de um método para gerenciamento de riscos integrado a todas as fases do ciclo de vida BPM com a utilização de ferramentas adequadas de apoio. A partir das constatações citadas, o método propõe a obtenção de processos customizados às necessidades do negócio e de seus usuários incluindo atividades relacionadas aos riscos identificados em sua aplicação. O tratamento de riscos passa a ser uma atividade rotineira da execução dos processos de negócio e a mudança cultural associada passa a acontecer gradativamente à medida que os processos vão sendo executados e os riscos tratados adequadamente.

A principal motivação para propor a integração de forma completa inclui: aumentar a consciência de riscos associados aos processos de negócios, melhorar a produtividade dos processos, otimizar recursos e fornecer condições adequadas de avaliação dos processos pelos seus responsáveis. Para avaliação do método, este foi aplicado em um processo real de uma organização conhecedora de BPM e riscos.

Este artigo está organizado da seguinte forma: na seção 2 é apresentado um referencial teórico necessário para um melhor entendimento do estudo. Na seção 3 são apresentados o método proposto, modelo geral e a aplicação do método. A seção 4 são apresentados os resultados e discussões, sendo detalhados as etapas e os resultados. Finalmente, na seção 5 são apresentadas as conclusões e recomendações para trabalhos futuros.

\section{Referencial teórico}

Esta seção descreve uma visão geral da fundamentação teórica relacionada a técnica de gerenciamento de processos de negócio - BPM, a técnica de gerenciamento de riscos e salienta a importância de um método para integração das técnicas.

\subsection{Gerenciamento de Processos de Negócio - BPM}

Assume-se, neste estudo, que "Um processo é um conjunto definido de atividades ou comportamentos executados por humanos ou máquinas para alcançar uma ou mais metas" (ABPMP, 2013, p. 67). Para Baldam (2014), a visão por processos procura entender o que precisa ser feito e qual a melhor forma de fazê-lo dentro de uma organização.

Para suportar essa interação organizacional dos processos surge o BPM que é uma abordagem disciplinada para identificar, desenhar, executar, documentar, medir, monitorar, controlar e melhorar os processos de negócios visando alcançar resultados consistentes e alinhados com os objetivos 
estratégicos da organização (ABPMP, 2013). O BPM demonstrou ser uma abordagem coesa para conferir a maturidade e agilidade das organizações que o aplicam (Dumas et al., 2018). Um processo de negócio é uma sequência de atividades dependente do tempo. A coordenação dessas atividades é necessária para a execução bem sucedida de leis e regulamentos. A execução de processos de negócios geralmente é suportada pelos sistemas de Gerenciamento de Processos de Negócios - BPM (Gong \& Janssen, 2012).

\subsection{Ciclo de vida BPM}

Existem diversos modelos propostos na literatura para orientar a gestão de processos de negócios sendo que a maioria deles assume a forma cíclica baseada no modelo PDCA (do inglês, Plan, Do, Check, Act) com as atividades organizadas em fases e se repetindo a cada fase, por esta razão falase em ciclos de vida BPM (Baldam, 2014).

De acordo com Oliveira (2014), o método Easy Business Process Management (EBPM) apresenta de forma objetiva e de fácil compreensão o ciclo de vida BPM. As fases poderão ser executadas na sequência apresentada de forma integral ou simplificada, de acordo com as necessidades do projeto de BPM a ser implementado. O mapeamento do processo atual é chamado de "AS IS" e o desenho do processo proposto ou futuro é chamado de "TO BE" alinhado com o conhecimento descrito no Common Body of Knowledge (CBOK) da ABPMP (2013), é também aderente ao ciclo PDCA.

\subsection{Gerenciamento de riscos}

Existem várias definições relacionadas ao risco. Exemplificado por Valeriano (1998), entende-se por risco a possibilidade de ocorrência de um resultado indesejável como consequência de qualquer evento. Porém, para a norma ISO 31000:2018 da Associação Brasileira de Normas Técnicas [ABNT] (2018), risco é o efeito da incerteza nos objetivos sendo que um efeito é um desvio em relação ao esperado. Pode ser positivo, negativo ou ambos, e pode criar ou resultar em oportunidades e ameaças.

A etimologia da palavra revela a sua origem no italiano antigo, risicare, cujo significado é "ousar". Portanto, sua conotação original implica não apenas prejuízo (compreensão usual), mas também ganho decorrente de uma decisão frente a uma oportunidade (Baldam, 2014). Assim, qualquer operação está sujeita a ter riscos associados. De acordo com a norma ISO 31000:2018, o gerenciamento de riscos consiste em atividades coordenadas para dirigir e controlar uma organização no que se refere a riscos (ABNT, 2018).

O principal objetivo do gerenciamento de riscos para as organizações é identificá-los e controlálos continuamente, uma vez que parte do princípio de que eles são dinâmicos, ou seja, podem emergir, mudar ou desaparecer à medida que os contextos externo e interno de uma organização mudam (Baldam, 2014, Chapman, 2011 e Kim et al., 2017). 
As primeiras propostas de modelo para integração do gerenciamento de processos de negócio ao gerenciamento de riscos surgiram com os trabalhos de Rosemann et al. (2005), Islam et al. (2009). Portanto, a necessidade da integração das duas técnicas motivou o desenvolvimento do gerenciamento de processos de negócio consciente dos riscos denominado: R-BPM (Suriadi et al., 2014, Haggag, Khedr, \& Montasser, 2015 e Ferreira, 2016). O estudo proposto por Lamine et al. (2020) demonstra uma estrutura integrada de gerenciamento de processos de negócio e gerenciamento de riscos relacionado ao modelo proposto por Rosemann et al. (2005) e utiliza um framework chamado BPRIM baseado no trabalho proposto por Sienou et al. (2010).

Apesar da tendência de as organizações indicarem um modelo específico de gerenciamento de riscos integrado ao ciclo de vida BPM, não existe uma forma única para a integração entre as duas técnicas. É importante que se faça uma análise dos objetivos a serem alcançados pela organização e definir o melhor modelo de integração. Para uma melhor análise da integração mais adequada para alcançarmos os objetivos deste trabalho, demonstramos no Quadro 1 a comparação e inter-relação das atividades de cada abordagem do gerenciamento de riscos com todas as fases do ciclo de vida BPM, mostrando suas similaridades.

\section{Quadro 1}

Comparação entre as principais abordagens de gerenciamento de riscos com as fases do ciclo de vida $B P M$

\begin{tabular}{|c|c|c|c|c|}
\hline \multirow{2}{*}{ Fases BPM } & \multicolumn{4}{|c|}{ Gerenciamento de riscos } \\
\hline & PMBOK, 2017 & ISO 31.0000:2018 & IBGC, 2017 & COSO, 2017 \\
\hline Planejamento & & $\begin{array}{l}\text { Escopo, contexto, } \\
\text { critério }\end{array}$ & & $\begin{array}{l}\text { Estratégia e definição de } \\
\text { objetivos }\end{array}$ \\
\hline \multirow{3}{*}{$\begin{array}{c}\text { Modelagem do } \\
\text { processo atual } \\
\text { (AS IS) } \\
\text { Análise do } \\
\text { processo } \\
\text { Desenho do } \\
\text { processo proposto } \\
\text { (TO BE) }\end{array}$} & $\begin{array}{l}\text { Identificação dos } \\
\text { riscos }\end{array}$ & $\begin{array}{l}\text { Identificação dos } \\
\text { riscos }\end{array}$ & $\begin{array}{l}\text { Identificar os } \\
\text { riscos }\end{array}$ & $\begin{array}{c}\text { Performance - } \\
\text { identificação de riscos }\end{array}$ \\
\hline & $\begin{array}{l}\text { Qualificação dos } \\
\text { riscos }\end{array}$ & Análise de riscos & $\begin{array}{l}\text { Classificar os } \\
\text { riscos }\end{array}$ & $\begin{array}{l}\text { Performance - avaliação e } \\
\text { priorização de riscos }\end{array}$ \\
\hline & $\begin{array}{l}\text { Desenvolvimento de } \\
\text { respostas a riscos }\end{array}$ & Avaliação de riscos & Avaliar os riscos & $\begin{array}{l}\text { Performance - visão de } \\
\text { portfólio }\end{array}$ \\
\hline Implementação & & $\begin{array}{l}\text { Tratamento de } \\
\text { riscos }\end{array}$ & $\begin{array}{c}\text { Implementar a } \\
\text { função de gestão } \\
\text { de riscos }\end{array}$ & Análise e revisão \\
\hline \multirow[t]{3}{*}{$\begin{array}{c}\text { Monitoramento e } \\
\text { controle }\end{array}$} & $\begin{array}{l}\text { Controle de } \\
\text { respostas a riscos }\end{array}$ & $\begin{array}{l}\text { Monitoramento e } \\
\text { análise crítica }\end{array}$ & $\begin{array}{l}\text { Estrutura de } \\
\text { controles } \\
\text { internos e } \\
\text { monitorar }\end{array}$ & Governança e cultura \\
\hline & & $\begin{array}{l}\text { Comunicação e } \\
\text { consulta }\end{array}$ & & $\begin{array}{l}\text { Informação, comunicação } \\
\text { e divulgação }\end{array}$ \\
\hline & & Registro e relato & & \\
\hline
\end{tabular}

Fonte: Adaptado de Ferreira (2016). 
Como resultado foi constatado que, apesar de suas particularidades, todos os modelos têm em comum as atividades de identificar, avaliar, tratar e monitorar os riscos e, como o gerenciamento de riscos deve ser realizado de forma cíclica, as atividades que são comuns a todo modelo de gerenciamento de riscos seguem um formato semelhante com as fases do ciclo de vida BPM, o qual se baseia no método iterativo PDCA.

A abordagem de gerenciamento de risco escolhida foi a NBR ISO 31000:2018 (Gestão de riscos - Princípios e diretrizes), seguindo os critérios de: melhor integração entre as etapas do gerenciamento de riscos e as fases do ciclo de vida BPM, aplicada a uma ampla gama de atividades, incluindo estratégias, decisões, operações, processos, funções, projetos, produtos, serviços e ativos e aplicada a qualquer tipo de risco, independentemente de sua natureza, quer tenha consequências positivas ou negativas.

\subsection{Trabalhos correlatos de integração}

A atividade inicial deste estudo compreendeu na realização de uma pesquisa bibliográfica dos principais trabalhos de integração do gerenciamento de riscos ao BPM e as suas lacunas. O Quadro 2 apresenta os trabalhos analisados. 
Quadro 2

Abordagens atuais dos principais trabalhos disponíveis na literatura

Trabalhos

Rosemann et al.

Islam et al.

(2009)

Cope et al

(2010)

Sienou et al.

(2010)

Conforti et al.
(2011)

Hofstede et al

Haggag, Khedr e Montasser

(2015)

Ferreira et al.

(2016)

Shah et al.

(2017)

Lamine et al.

(2020)

\section{Pontos relevantes}

Utiliza técnicas para identificar os riscos para os processos de negócios através de um método processual.

Utiliza técnicas para identificar os riscos para os processos de negócios utilizando modelos organizacionais.

Utiliza a ferramenta de BPMN para representar informações relacionadas a identificação dos riscos.

Utiliza técnicas para identificar os riscos para os processos de negócios utilizado um framework chamado Business Process Risk Management Integrated Method (BPRIM).

Utiliza técnicas para identificar e analisar riscos em processos de negócios na fase de execução.

Utiliza técnicas de integração que permite a identificação, análise e monitoramento dos riscos nos processos.

Utiliza técnicas e propõem um modelo para identificar riscos em todas as fases do ciclo de vida BPM.

\section{Apresenta o modelo R-BPM,}

Gerenciamento de Processos de Negócios

\section{Consciente dos Riscos, que compara} existência ou não de atividades de gestão de riscos em cada fase do ciclo de vida BPM.

\section{Apresenta o modelo R-BPM,}

Gerenciamento de Processos de Negócios Consciente dos Riscos, com aplicabilidade prática.

Apresenta uma estrutura integrada de gerenciamento de processos de negócio e gerenciamento de riscos chamado Business Process Risk Management Integrated Method (BPRIM).

\section{Lacunas}

Utiliza apenas as fases de Modelagem (AS IS) no ciclo de vida BPM.

Utiliza apenas as fases de Modelagem (AS IS),

Análise e Desenho (TO BE) no ciclo de vida BPM.

Utiliza apenas as fases de Modelagem (AS IS), Análise e Desenho (TO BE) no ciclo de vida BPM. Não possui aplicabilidade prática.

Utiliza apenas as fases de Modelagem (AS IS) e Análise no ciclo de vida BPM. Não possui aplicabilidade prática.

Utiliza as fases de Modelagem (AS IS), Desenho (TO BE) e Implementação no ciclo de vida BPM. Porém, sem as fases de Planejamento, Analise e Monitoramento.

Utiliza apenas a fase de Modelagem (AS IS) no ciclo de vida BPM. Não possui aplicabilidade prática.

Utiliza as fases de Modelagem (AS IS), Análise, Desenho (TO BE), Implementação e Monitoramento no ciclo de vida BPM. Porém, sem a fase de planejamento.

Utiliza o modelo COSO: Enterprise Risk

Management - Integrated Framework para gerenciamento de riscos com enfoque aos riscos corporativos.

Utiliza as fases de Modelagem (AS IS), Desenho (TO BE) e Implementação no ciclo de vida BPM. Porém, não possui uma ferramenta de apoio.

Não utiliza todas as fases ciclo de vida BPM. Os autores pretendem aprimorar o estágio de execução aumentando recursos de simulação na ferramenta utilizada (ADOBPRIM).

Fonte: Elaborado pela autora.

Após análises efetuadas através dos trabalhos descritos no Quadro 2, é possível verificar que a principal lacuna identificada é a integração incompleta das abordagens de gerenciamento de riscos ao contexto de BPM. Evidenciamos também que o número de trabalhos disponíveis é reduzido e, em sua maioria, possuem pouca aplicabilidade prática, ausência de uma ferramenta de apoio adequada e 
utilizam modelos para tipos específicos de riscos. Este artigo visa propor um modelo suportado pelo trabalho de Ferreira (2016) para: integrar completamente o gerenciamento de riscos as fases do ciclo de vida BPM, apresentar resultados da aplicabilidade prática utilizando ferramentas de apoio adequadas e utilizar em qualquer tipo de risco. Sendo evidenciado através do método proposto, descrito a seguir.

\section{Estratégia de concepção}

Esta seção descreve o desenvolvimento do método proposto, a estruturação do modelo geral demonstrando a integração entre o gerenciamento de riscos e o BPM de forma iterativa e a aplicação em um processo real para avaliação do método.

\subsection{Método proposto}

Para Suriadi et al. (2014), com a utilização de técnicas consistentes de gerenciamento de riscos não é preciso perder tempo propondo novas técnicas de análise de riscos, em vez disso, pode-se concentrar no desenvolvimento de métodos que permitam a aplicação e adequação dessas técnicas de forma integrada em todas fases do ciclo de vida BPM.

O método possui um objetivo exploratório com uma abordagem de pesquisa-ação e foi desenvolvido com base na metodologia R-BPM demonstrada por Ferreira (2016) e nas análises dos trabalhos existentes na literatura, conforme evidenciado no Quadro 2, onde foi identificado os pontos mais relevantes e as principais lacunas de cada um dos estudos.

Em seguida, foi elaborado um modelo integrando as atividades do gerenciamento de riscos com a funcionalidade do ciclo de vida BPM, assumindo a forma iterativa, sendo suportado pelo ciclo PDCA Modelo geral (Figura 2).

Para validar o Modelo geral, foi realizada uma aplicação prática em uma organização que adota o gerenciamento de riscos e o BPM de forma básica e separada com a finalidade de atender os requisitos de seus clientes. O processo interno escolhido foi o recebimento físico e fiscal de materiais, tendo seus resultados insatisfatórios em auditorias internas realizadas anteriormente pela organização. A equipe multidisciplinar foi escolhida de forma estratégica e intencional, tendo sido selecionados especialistas das áreas envolvidas engajados nas técnicas o que possibilitou a criação de um ambiente propício às discussões.

Vale ressaltar que o engajamento da alta direção da organização, em todas as etapas de aplicação do método, foi de suma importância para a eficácia dos resultados.

A Figura 1 demonstra o passo a passo do método, em duas etapas, sendo o fluxo desenhado em BPMN (do inglês, Business Process Management Notation) que é um padrão criado em 2003 pela 
BPMI (do inglês, Business Process Management Initiative), hoje OMG (do inglês, Object Management Group), descrito por Oliveira (2014), para facilitar o entendimento.

Figura 1

Desenvolvimento do Método

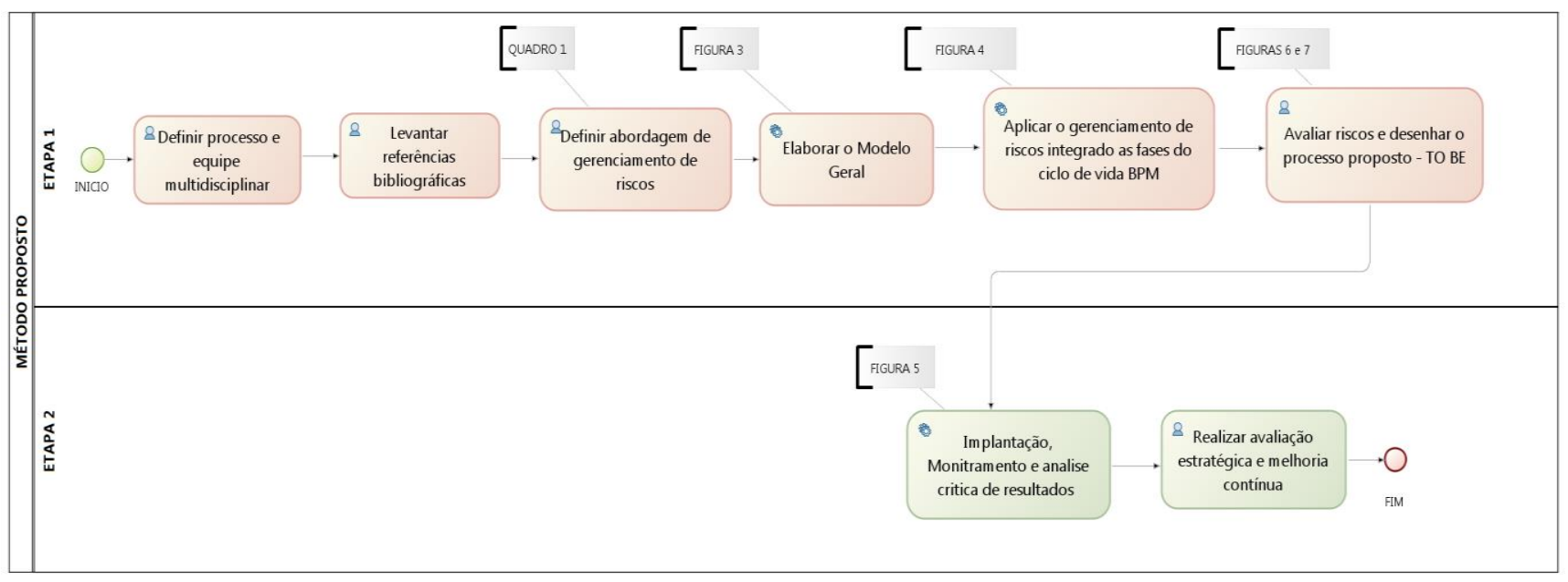

Fase Planejar - plan do ciclo PDCA

Fases de Fazer - do, Checar - check e Agir - act do ciclo PDCA

Fonte: Elaborada pela autora

\subsection{Modelo geral}

O modelo geral está representado pela Figura 2 e possui o mesmo funcionamento do ciclo de vida BPM, assumindo a forma cíclica do PDCA com as atividades organizadas nas etapas demonstradas pela Figura 1. Visando a identificar a origem das fases, foram evidenciadas, na cor branca, as fases do ciclo de vida BPM, propostas por Oliveira (2014); em cinza, as atividades da gestão de riscos da norma NBR ISO 31000:2018; e as letras coloridas, em destaque, evidenciam o ciclo PDCA. 
Figura 2

\section{Modelo geral}

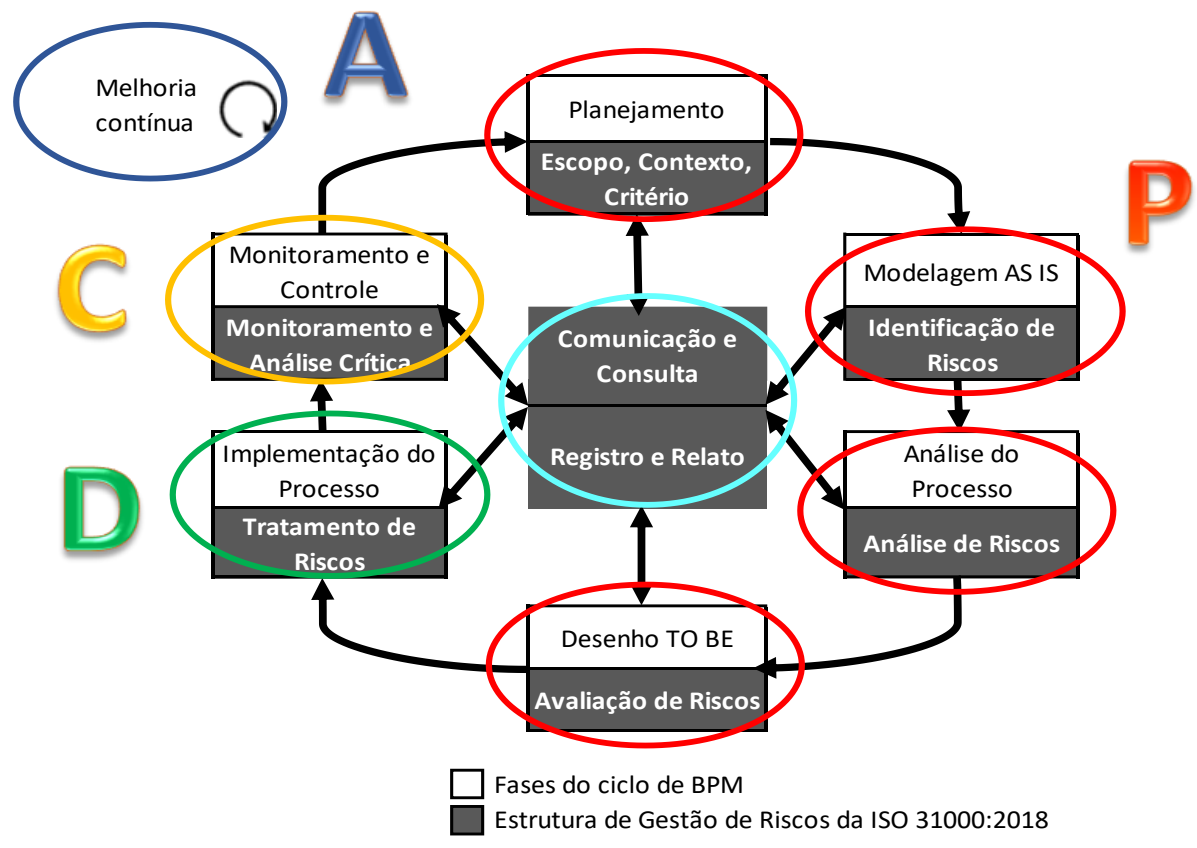

Fonte: Adaptado de Oliveira (2014) e Ferreira (2016).

A seguir serão detalhadas as interações efetuadas entre o desenvolvimento do método e seus documentos associados (Figura 1) com as fases integradas no modelo geral (Figura 2).

> Fase Planejar - plan do ciclo PDCA - Etapa 1 do planejamento, análise e definição do processo proposto

A Etapa 1 é a responsável por determinar o planejamento, a análise das fases do ciclo de vida BPM com os riscos associados, conforme requisitos da NBR ISO 31000:2018 e por fim, definir o processo proposto (TO BE) baseado na avaliação dos riscos identificados.

O núcleo central desta etapa é composto por um conjunto de quatro integrações: Planejamento e Escopo, contexto, critério; Modelagem AS IS e Identificação de riscos; Análise de processo e Análise de riscos e, por fim, Desenho TO BE e Avaliação de riscos. As ferramentas de apoio utilizadas são: o Excel, que permitiu inserir informações e compilar dados para tomadas de decisões da alta direção e o software Bonita Soft ${ }^{\circledast}$ (BONITA-SOFT, 2018), que possibilitou produzir a modelagem AS IS e desenho TO BE usando a notação BPMN a fim de estruturar o processo atual e proposto da organização.

A integração da fase de planejamento e escopo, contexto e critério consiste em alinhar a técnica do BPM com a estratégia da organização para desenvolver uma visão geral do processo a ser trabalhado. Essa etapa consiste em reuniões semanais com o grupo multidiciplinar, utilização dos dados existentes 
da organização, planejamento estratégico, requisitos de clientes, inspeções periódicas in loco para análise de atividades mapeadas e documentos/registros do processo.

A integração entre a fase de modelagem AS IS e identificação de riscos tem o objetivo de levantar informações e retratar o processo atual. Além disso, visa entender como o processo funciona e identificar as ameaças (risco negativo) e oportunidades (risco positivo) que podem afetar o processo. O principal desafio a ser alcançado pelo grupo é modelar o processo como realmente é, pois as informações são iniciais nesta fase.

Após a modelagem AS IS e identificação de riscos, efetua-se a integração da análise de processo e a de riscos, que possui como objetivo a identificação dos problemas no processo atual e suas causas. Assim, analisam-se as incertezas de forma a considerar a probabilidade de ocorrência e a severidade do impacto gerado. O principal desafio a ser alcançado pelo grupo é verificar, de forma qualitativa, os riscos e suas causas, levando em consideração a opinião dos especialistas do grupo multidisciplinar.

Após as análises de processo e riscos, efetua-se a integração do desenho do processo proposto (TO BE) e a avaliação de riscos, que possui como objetivo propor mudanças que visam à padronização dos processos e considerar até que nível os riscos podem impactar na realização dos objetivos estratégicos da organização, isso se obtém com base em duas perspectivas: probabilidade e impacto. Nessa fase, avalia-se se o risco será tratado ou não naquele momento. O principal desafio a ser alcançado pelo grupo é propor mudanças eficazes baseadas no processo atual.

É possível que sejam identificados riscos negativos - ameaças ou riscos positivos -, oportunidades que não foram identificadas anteriormente. Assim, é importante que antes de avaliar novos riscos retorne-se à Etapa 1 para registrá-los no Mapa de gerenciamento de riscos (Figura 3).

As fases de Fazer - do, Checar - check e Agir - act do ciclo PDCA - Etapa 2 da aplicação e melhoria contínua

A Etapa 2 visa à aplicação prática e melhoria contínua das fases de planejamento, análise e definição do processo proposto descritos na Etapa 1. Utiliza a compilação e apresentação dos resultados para uma melhor tomada de decisão da alta direção da organização. Essa etapa teve reuniões semanais com o grupo multidisciplinar e com as partes interessadas, ou seja, pessoas envolvidas no processo e responsáveis por atuar na eficácia das ações propostas no plano de tratamento de riscos e na melhoria contínua do método.

A fase Fazer - do possui como objetivo principal a operacionalização da fase anterior - processo proposto TO BE. É a integração entre a implementação do processo e tratamento de riscos, que visa à implantação de procedimentos novos ou revisados, a definição sobre a implementação total ou parcial do processo proposto TO BE e a elaboração do plano de tratamento de riscos com ações corretivas e/ou 
preventivas necessárias, definição dos responsáveis e do planejamento de prazos. Além disso, o plano deve conter ações necessárias para assegurar que as respostas aos riscos sejam executadas em caso de concretização de algum risco durante a execução do processo.

A fase Checar - check é a integração entre o monitoramento e controle do processo com monitoramento e análise crítica de riscos e tem como objetivo monitorar o avanço das ações propostas no plano de tratamento de riscos, verificar o que realmente mudou no processo, realizar medições de desempenho do processo proposto (TO BE) e analisar a conformidade visando à melhoria contínua do processo trabalhado.

Ao final de cada ciclo, após a fase de monitoramento e controle do processo, é realizada uma análise da iteração antes de se iniciar um novo ciclo, em um processo chamado de melhoria contínua, na fase Agir - act. Assim, o modelo geral proposto será aprimorado através das avaliações realizadas pelo grupo multidisciplinar.

Os requisitos de Comunicação e consulta e Registro e relato foram integrados e inseridos no centro do modelo geral, pois estes interagem continuamente com as demais fases do ciclo de vida BPM e com os requisitos da NBR ISO 31000:2018. Além de servir como divulgação da informação, os registros são base para lição aprendida de novas aplicações do modelo, e a comunicação contribui para que o ambiente corporativo reflita os valores e a cultura de riscos desejada pela organização (IBGC, 2017).

\subsection{Documentos associados}

A partir da definição do desenvolvimento do método (Figura 1) e da estruturação do modelo geral (Figura 2) serão demonstrados de forma ilustrativa, a seguir, os documentos associados que suportam cada fase integrada.

Com base na análise efetuada das principais abordagens de gerenciamento de riscos com as fases do ciclo de vida BPM - Quadro 1, foram definidos os critérios de planejamento, de escopo, de contexto e de critério em conformidade com os requisitos do ciclo de vida BPM e da NBR ISO 31000:2018, ou seja, foi definido o que se deseja alcançar e como isso será feito.

A fase integrada de modelagem (AS IS) e identificação de riscos foi integrada com objetivo levantar informações, entender como o processo funciona atualmente e identificar as ameaças (risco negativo) e oportunidades (risco positivo) associadas. Todos os riscos levantados foram identificados por número sequencial e registrados no campo "ID" sendo destacado no Mapa de gerenciamento de riscos - Figura 3. Como forma de conexão e rastreabilidade, cada risco registrado no campo ("ID") da Figura 3 estão dispostos na Matriz de priorização das Figuras 4 e 5 deste artigo.

A fase de análise do processo foi integrada ao requisito de análise de riscos, pois, nessa fase, define-se o que é mais importante, priorizando os riscos de forma a considerar a probabilidade de 
ocorrência e a severidade do impacto gerado a serem tratados de forma eficaz. Os Riscos com grau "Alto" são evidenciados em vermelho, os de grau "Médio" são evidenciados em laranja e os de grau "Baixo" são evidenciados em amarelo, conforme indicado no campo "Grau do Risco" da Figura 3 - Mapa de gerenciamento de riscos.

Figura 3

Mapa de gerenciamento de riscos - identificação e análise

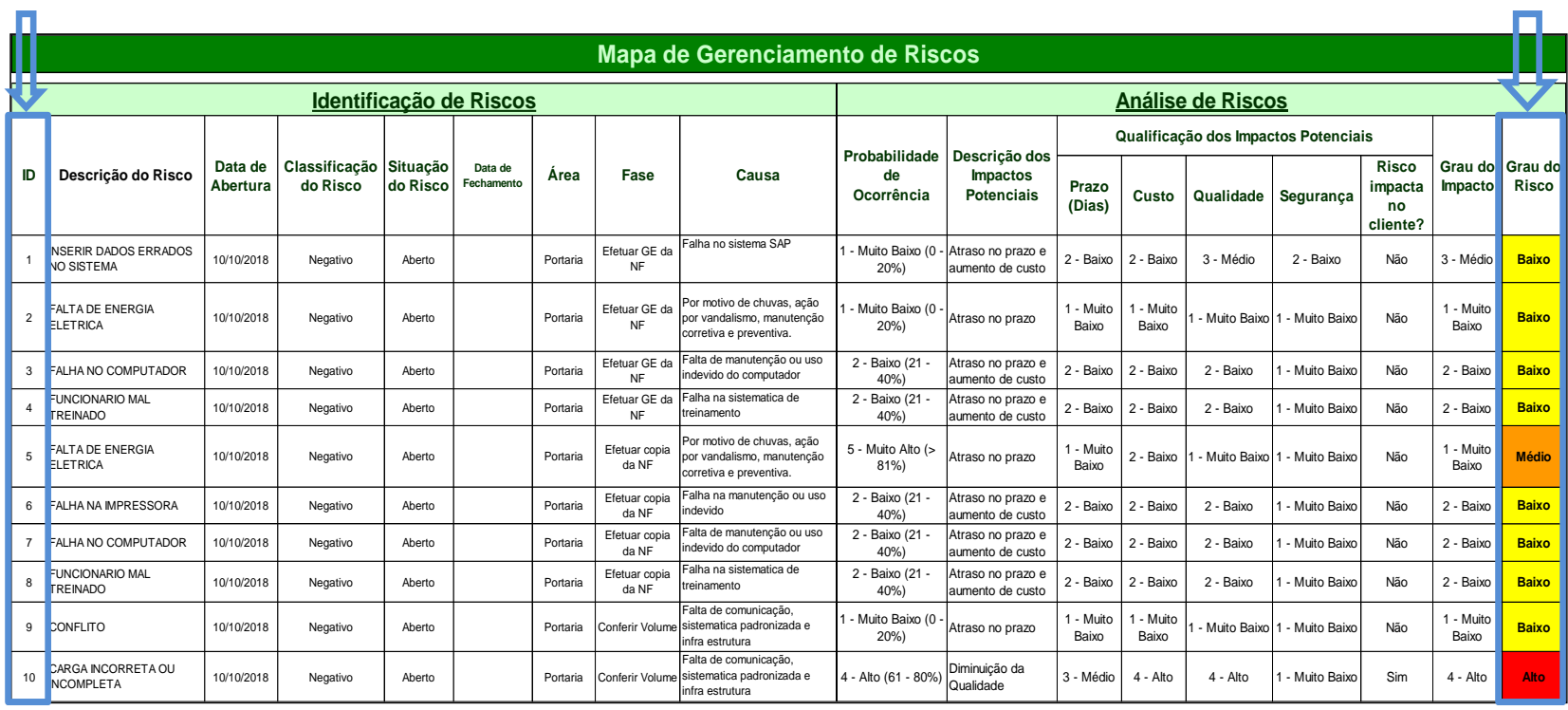

Fonte: Elaborada pela autora.

A fase de desenho do processo proposto ( $T \mathrm{O} \mathrm{BE}$ ) e o requisito de avaliação de riscos foram integrados, pois, nessa fase, realiza-se a verificação de quais riscos associados ao processo serão tratados ou não naquele momento. Nessa fase é efetuado o desenho do processo proposto - TO BE em BPMN sendo validado pela equipe multidisciplinar e partes interessadas antes de sua implementação.

A matriz de priorização de riscos foi utilizada como técnica para avaliação tanto para o processo atual (AS IS), conforme Figura 4, quanto para o processo proposto (TO BE), conforme Figura 5 e os respectivos resultados de sua aplicação serão descritos na seção 4. As combinações de probabilidade e impacto resultam em uma classificação de grau do risco alto, médio e baixo e proporcionam às organizações a possibilidade de visualizar os riscos que deverão ser tratados inicialmente, uma vez que afetam de forma crítica os objetivos estratégicos e impactam severamente os processos.

A fase de implementação do processo foi integrada ao tratamento de riscos, pois elabora-se um plano de tratamento de riscos, conforme processo atual (AS IS). O plano deve descrever as ações corretivas e/ou as de melhoria que asseguram o tratamento pontuado como riscos críticos de acordo com a tolerância e com o nível de aceitação à riscos absorvido pela organização. Ainda nessa etapa é 
definido o método de implementação, ou seja, a verificação se o processo proposto substituirá totalmente o atual ou se poderão funcionar em paralelo até a validação do processo proposto - TO BE.

A fase de monitoramento e controle do processo foi integrada ao monitoramento e análise crítica de riscos e tem como objetivo controlar o avanço das ações propostas no plano de tratamento de riscos, verificar o que realmente mudou no processo, realizar medições de desempenho do processo proposto (TO BE) e analisar a conformidade visando à melhoria contínua do processo trabalhado.

\section{Resultados}

Esta seção tem o objetivo de descrever os resultados obtidos com a aplicação prática do método proposto no processo real de uma organização, no qual se analisou as percepções da equipe multidisciplinar e partes interessadas das áreas de riscos e de BPM utilizando o modelo geral e as ferramentas de apoio.

\subsection{Comparação com os trabalhos correlatos}

Os critérios descritos na seção 3 foram baseados nos trabalhos correlatos existentes na literatura e apresentados na Seção 2.4. Os resultados obtidos pelo método proposto permitiram comprovar que: as atividades do gerenciamento de riscos podem ser integradas a todas as fases do ciclo de vida BPM, a aplicabilidade prática melhora o resultado do processo produtivo (comparação entre o processo atual - AS IS e o processo proposto TO BE que serão apresentados a seguir) e com a adoção da abordagem de gerenciamento de riscos pela NBR ISO 31000 pode-se utilizar o método em qualquer tipo de risco. A Quadro 3 mostra o resultado da comparação entre o método proposto e os trabalhos correlatos na literatura. 


\section{Quadro 3}

Comparação entre trabalhos correlatos e método proposto

\begin{tabular}{|c|c|c|c|c|c|c|c|c|c|c|c|c|c|c|}
\hline \multirow[b]{2}{*}{$\begin{array}{l}\text { Trabalhos } \\
\text { correlatos }\end{array}$} & \multicolumn{6}{|c|}{ Gerenciamento de riscos } & \multirow[b]{2}{*}{ Abordagem } & \multicolumn{6}{|c|}{ Fases do ciclo de vida BPM } & \multirow[b]{2}{*}{$\begin{array}{c}\text { Aplicabilidade } \\
\text { prática }\end{array}$} \\
\hline & $\begin{array}{c}\text { Escopo, } \\
\text { contexto, } \\
\text { critério }\end{array}$ & Identificação & Análise & Avaliação & Tratamento & $\begin{array}{c}\text { Monitoramento e } \\
\text { Análise crítica }\end{array}$ & & Planejamento & $\begin{array}{l}\text { Modelagem } \\
\text { AS IS }\end{array}$ & Análise & $\begin{array}{c}\text { Desenho TO } \\
\text { BE }\end{array}$ & Implementação & $\begin{array}{c}\text { Monitoramento } \\
\text { e controle }\end{array}$ & \\
\hline $\begin{array}{l}\text { Rosemann et al. } \\
\text { (2005) }\end{array}$ & & $\mathrm{x}$ & & & & & - & & $\mathrm{x}$ & & & & & - \\
\hline Islam et al. (2009) & & $\mathrm{x}$ & $\mathrm{x}$ & & & & - & & $\mathrm{x}$ & $\mathrm{x}$ & $\mathrm{x}$ & & & $\mathrm{x}$ \\
\hline Cope et al. (2010) & & $\mathrm{x}$ & $\mathrm{x}$ & & & & $\begin{array}{c}\text { COSO } \\
\text { Riscos } \\
\text { corporativos }\end{array}$ & & $\mathrm{x}$ & $\mathrm{x}$ & $\mathrm{x}$ & & & - \\
\hline Sienou et al. (2010) & & $\mathrm{x}$ & $\mathrm{x}$ & & & & $\begin{array}{c}\text { COSO } \\
\text { Riscos } \\
\text { corporativos }\end{array}$ & & $\mathrm{x}$ & & & & & - \\
\hline $\begin{array}{l}\text { Conforti et al. } \\
\text { (2011) }\end{array}$ & & $\mathrm{x}$ & $\mathrm{x}$ & & & & - & & $\mathrm{x}$ & & $\mathrm{x}$ & $\mathrm{x}$ & & $\mathrm{x}$ \\
\hline $\begin{array}{l}\text { Hofstede et al. } \\
\text { (2011) }\end{array}$ & & $\mathrm{x}$ & $\mathrm{x}$ & & & & - & & $\mathrm{x}$ & & & & & - \\
\hline Haggag et al. (2015) & & $\mathrm{x}$ & & & & & - & & $\mathrm{x}$ & $\mathrm{x}$ & $\mathrm{x}$ & $\mathrm{x}$ & $\mathrm{x}$ & $\mathrm{x}$ \\
\hline $\begin{array}{l}\text { Ferreira et al. } \\
\text { (2016) }\end{array}$ & & $\mathrm{x}$ & $\mathrm{x}$ & $\mathrm{x}$ & $\mathrm{x}$ & $\mathrm{x}$ & $\begin{array}{c}\text { Coso } \\
\text { Riscos } \\
\text { corporativos }\end{array}$ & $\mathrm{x}$ & $\mathrm{x}$ & $\mathrm{x}$ & $\mathrm{x}$ & $\mathrm{x}$ & $\mathrm{x}$ & $\mathrm{x}$ \\
\hline $\begin{array}{l}\text { Shah et al. } \\
(2017)\end{array}$ & & $\mathrm{x}$ & $\mathrm{x}$ & $\mathrm{x}$ & $\mathrm{x}$ & $\mathrm{x}$ & - & $\mathrm{x}$ & $\mathrm{x}$ & $\mathrm{x}$ & $\mathrm{x}$ & $\mathrm{x}$ & & $\mathrm{x}$ \\
\hline $\begin{array}{l}\text { Lamine et al. } \\
\text { (2020) }\end{array}$ & & $\mathrm{x}$ & $\mathrm{x}$ & $\mathrm{x}$ & $\mathrm{x}$ & $\mathrm{x}$ & $\begin{array}{c}\text { NBR ISO } 31000 \\
\text { Riscos gerais }\end{array}$ & $\mathrm{x}$ & $\mathrm{x}$ & $\mathrm{x}$ & & & & $\mathrm{x}$ \\
\hline $\begin{array}{l}\text { Método proposto } \\
\text { (2019) }\end{array}$ & $\mathbf{x}$ & $\mathbf{x}$ & $\mathbf{x}$ & $\mathbf{x}$ & $\mathbf{x}$ & $\mathbf{x}$ & \begin{tabular}{|c|} 
NBR ISO \\
31000 \\
Riscos gerais
\end{tabular} & $\mathbf{x}$ & $\mathbf{x}$ & $\mathbf{x}$ & $\mathbf{x}$ & $\mathbf{x}$ & $\mathbf{x}$ & $\mathbf{x}$ \\
\hline
\end{tabular}

Fonte: Elaborada pela autora.

\subsection{A fase planejar - plan do ciclo PDCA}

Esta fase iniciou-se com a análise da literatura, evidenciada na seção 4.1, as definições necessárias referentes ao processo trabalhado e a abordagem de gerenciamento de riscos escolhida (Quadro 1). Após, foi efetuada a modelagem fim a fim do processo atual (AS IS) do processo escolhido obtendo-se como resultado a execução das atividades de forma sistêmica e mais unificada possível; assim, o que era executado em duplicidade e separado passou a ser executado de uma vez, de forma integrada e ordenada.

A atividade de identificação e análise de riscos utilizou o mapa de gerenciamento de riscos como registro, conforme a Figura 3. Nessa fase, todos os riscos foram considerados "abertos", pois, até então, nenhum havia sido tratado e, até a finalização dessa fase, nenhum risco havia sido eliminado ou cancelado.

Como resultado, foram identificados 400 riscos, tendo sido utilizada a matriz de priorização de riscos como técnica para evidenciar a distribuição dos riscos, os quais foram classificados pelos seus respectivos graus. O tratamento dos riscos deve ser desenvolvido começando com os riscos negativos - ameaças encontradas no quadrante superior direito (os riscos críticos), que concentra riscos de 
probabilidade muito alto e impacto muito alto, ou seja, de alta severidade. À medida que o seu grau de severidade diminui de intensidade, os riscos podem ser monitorados e tratados com periodicidade mais espaçada (IBGC, 2017). Consequentemente, os riscos classificados como críticos no processo atual (AS IS) devem ser priorizados, conforme demonstrado na Figura 4.

\section{Figura 4}

Matriz de priorização de riscos (Probabilidade X Impacto) - processo atual AS IS

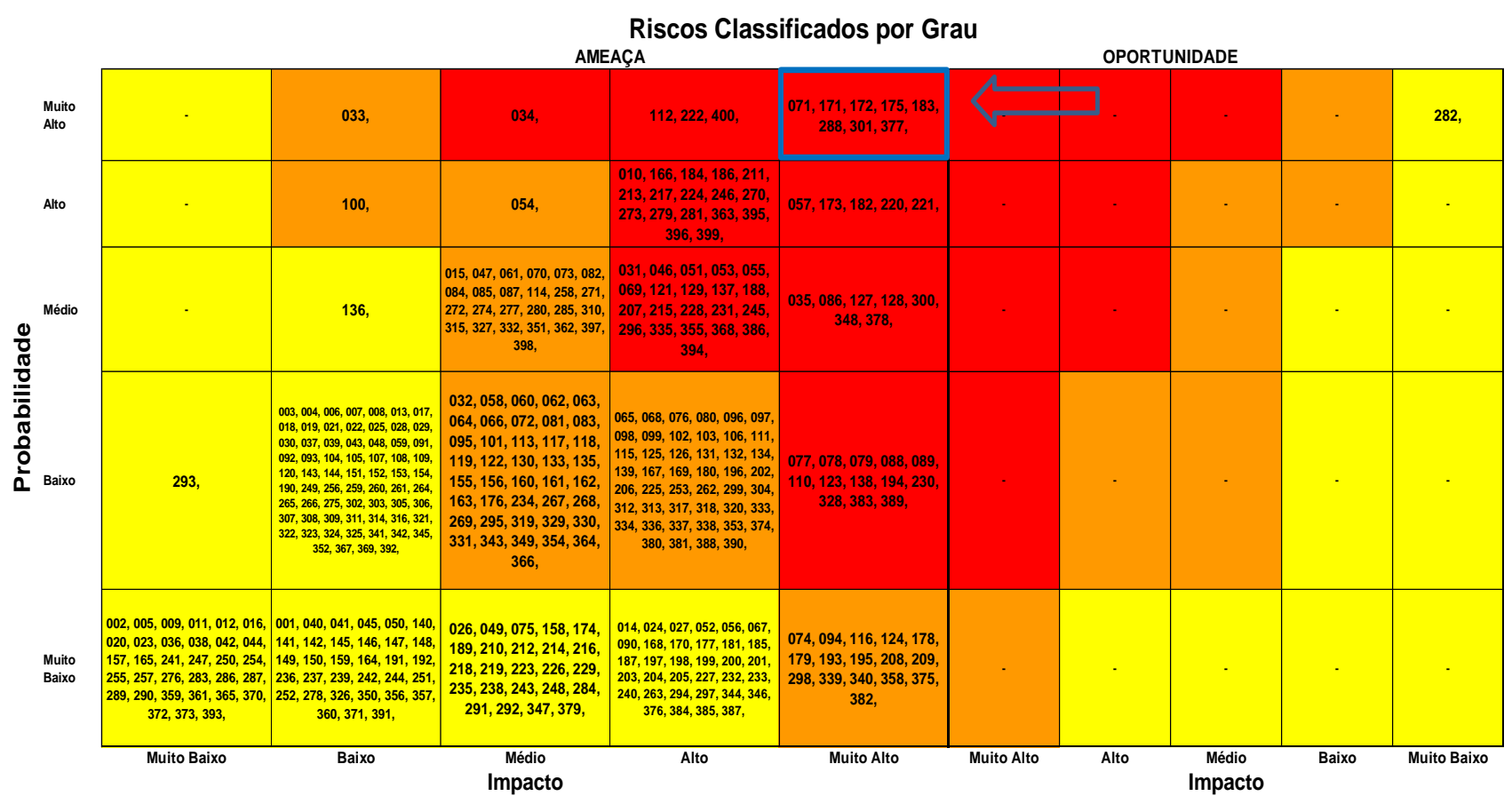

Fonte: Elaborada pela autora.

Nesta fase, foram avaliados 75 riscos altos, sendo que oito destes foram classificados, de acordo com a matriz de priorização, como muito altos, ou seja, devem ser tratados e verificados juntamente com os responsáveis dos processos, as ações com previsibilidade para curto prazo e com uma adequação de recursos para maior agilidade das ações.

Após a fase de tratamento dos riscos e implementação do processo, os oito riscos críticos identificados na matriz de priorização de riscos do processo atual AS IS foram alterados ou por eliminação ou por redução dos impactos potenciais, passando para uma classificação de grau mais baixo, conforme demonstrado na matriz de priorização de riscos do processo proposto TO BE (Figura 5). 
Figura 5

Matriz de priorização de riscos (probabilidade $\mathrm{x}$ impacto) - processo proposto TO BE

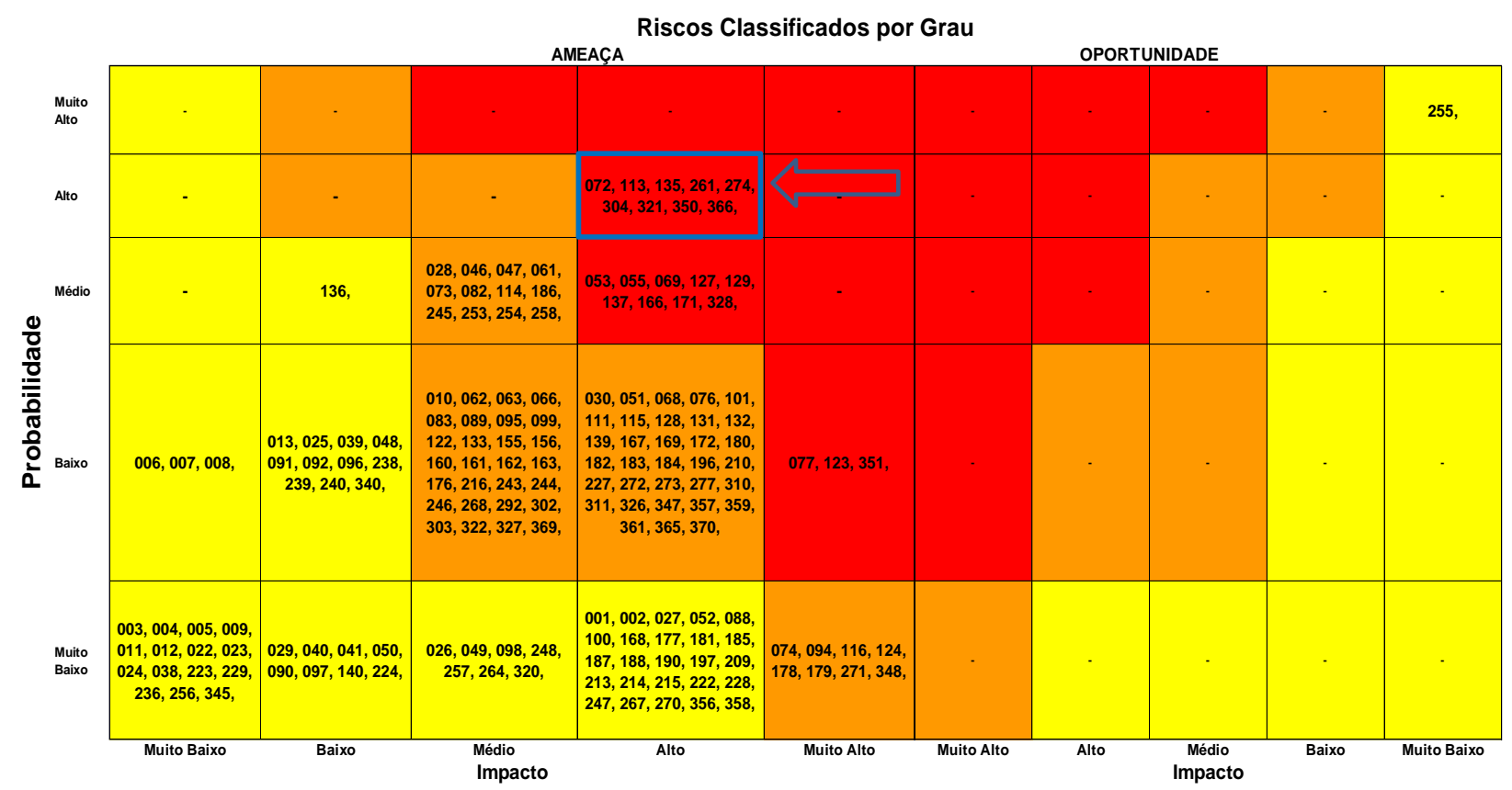

Fonte: Elaborada pela autora.

Como se pode verificar, foram avaliados 21 riscos altos, sendo que nove destes foram classificados, de acordo com a matriz de priorização (Figura 5), como altos, ou seja, esses riscos devem ser reportados à gerência do processo da organização para serem tratados em curto prazo.

Foi mapeado, inicialmente, um total de 400 riscos; desse total, 67 riscos foram eliminados, porém, foram inseridos 37 riscos novos, permanecendo um total de 370 riscos para o processo proposto TO BE. Desse total, permaneceram 173 riscos abertos, sendo 172 riscos classificados como ameaças e um como oportunidade, tendo sido fechados 197 riscos. Verificou-se, também, que 233 riscos impactaram diretamente no atendimento aos requisitos dos clientes no processo atual e, no processo proposto, esse número reduziu para 114, porém, todos os riscos foram classificados como ameaças.

\subsection{As fases do, check e act para o ciclo PDCA}

A fase Do - Fazer foi iniciada com a separação o tratamento dos oito riscos classificados como críticos, ou seja, esses riscos foram reportados à alta direção da organização, juntamente com a análise da implantação do processo proposto TO BE, para serem tratados com a maior agilidade possível.

A fase Check - Checar/Verificar foi direcionada conforme o plano de tratamento de riscos onde foram elaboradas ações de resposta aos riscos, determinados os responsáveis e os executores das ações, a fim de monitorar, controlar e verificar a eficácia das ações, com evidências.

Por fim, a fase Act - Agir foi demonstrada com base no comparativo dos impactos potenciais (riscos que afetam diretamente aos requisitos dos clientes) classificados como "muito alto" entre o 
processo atual AS IS e o processo proposto TO BE, após a aplicação do modelo proposto, onde foi obtida uma redução de $51,1 \%$ no prazo, $22,2 \%$ no custo, $49,4 \%$ na qualidade e $20 \%$ na segurança.

Os resultados foram analisados pela equipe multidisciplinar e partes interessadas, sendo proposto, como melhoria contínua, a aplicação do método em um novo ciclo para tratamento dos nove riscos classificados como altos (Figura 5).

\section{Conclusões}

A adoção do gerenciamento de processos de negócio - BPM é de crescente importância, uma vez que o mercado está cada vez mais competitivo e imprevisível. A integração do gerenciamento de processos de negócios ao gerenciamento de risco (R-BPM) é necessária para melhorar o desempenho e a robustez dos processos e, consequentemente, aumentar a produtividade.

As pesquisas realizadas nos poucos trabalhos disponíveis na literatura revelaram a fragilidade de estudos sobre o R-BPM em relação a integração completa, os fundamentos teóricos necessários para elaboração da estrutura, as ferramentas adequadas de apoio e a abrangência da aplicabilidade prática para cientistas e profissionais da área. A principal dificuldade de o processo ser flexível e ágil é causada pela complexidade de implementação da gestão de processos de negócio, incluindo as incertezas sobre o que é preciso adaptar, as muitas partes interessadas, as negociações entre as opções de implementação, a falta de visão geral sobre os processos, a dificuldade nos fluxos de informações e a falta de conhecimento sobre como conseguir flexibilidade e agilidade (Gong \& Janssen, 2012). Portanto, este estudo foi motivado por essas lacunas.

Nossa principal contribuição consiste em apresentar um método com resultados satisfatórios da aplicação prática realizada em qualquer tipo de organização e com qualquer tipo de risco associado com o objetivo de melhorar a produtividade. Para tal, elaborou-se um modelo geral que permitiu integrar o gerenciamento de riscos com todas as fases do gerenciamento de processos de negócios de forma suportada ao ciclo de vida BPM.

O modelo geral utilizou a estrutura de gerenciamento de riscos da NBR ISO 31000:2018 e o ciclo de vida BPM proposto por Oliveira (2014) de forma iterativa com a finalidade de facilitar o entendimento do método proposto, proporcionar maior agilidade nos resultados obtidos e flexibilizar sua aplicação, pois os riscos e os processos são mutantes.

A avaliação mostrou, através dos resultados da seção 4, que o método proposto, neste artigo, aumentou a produtividade do processo trabalhado, sendo que:

- Agregou um melhor aproveitamento de recursos, visto que algumas atividades dos processos envolvidos foram unificadas ou até mesmo eliminadas; 
- Proporcionou um melhor atendimento aos requisitos dos clientes, pois houve uma redução nos impactos potenciais (prazo, custo, qualidade e segurança);

- Reduziu a quantidade de riscos "altos" e "muito altos" distribuídos por área, evitando, assim, as possíveis ameaças aos objetivos estratégicos da organização;

- Aumentou a agilidade na identificação, na análise, na avaliação e no tratamento dos riscos através da visibilidade e interação entre os processos e seus riscos associados;

- Direcionou os riscos identificados aos devidos responsáveis.

Portanto, é importante salientar que é necessário efetuar uma análise dos resultados a cada execução do ciclo do modelo geral na Etapa 2 - Melhoria continua para aprimoramento do método.

Recomendamos, para trabalhos futuros, a adoção de técnicas e ferramentas para uma análise quantitativa de riscos, já que para este trabalho foram utilizadas técnicas e ferramentas qualitativas em função de atender os objetivos propostos. A análise quantitativa sempre nos fornecerá um universo mais consistente de informações. A análise qualitativa, por sua vez, é mais rápida, exige menos tempo e recursos e, dependendo da habilidade da equipe, pode ser muito eficaz (Hall, 2001).

Por fim, uma questão que pode ser melhorada é a realização de pesquisas semelhantes em outras organizações, tanto públicas como privadas. Desta forma será possível aprimorar a aplicabilidade do método proposto aqui em diferentes organizações.

\section{Referências}

Associação Brasileira de Normas Técnicas. ABNT. (2012). NBR ISO 31010: Gestão de riscos - Técnicas para o processo de avaliação de riscos. Rio de Janeiro: ABNT.

Associação Brasileira de Normas Técnicas. ABNT. (2018). NBR ISO 31000: Gestão de riscos - Princípios e diretrizes. Rio de Janeiro: ABNT.

Association of Business Process Management Professionals. ABPM. (2013). Guide for business process management: common body of knowledge. ABPMP.

Baldam, R. L. (2014). Gerenciamento de processos de negócios - BPM: uma referência para implantação prática. Rio de Janeiro: Elsevier.

BONITASOFT. (2018). Bonita BPM. Disponível em: http://www.bonitasoft.com. Acesso em: 14 de outubro. 2019.

Chapman, R. J. (2011). Simple Tools and Techniques for Enterprise Risk Management, John Wiley \& Sons, Chichester, 2 nd edition edition.

Committee of Sponsoring Organizations of the Treadway Commission. COSO. (2017). Enterprise Risk Management: Integrated Framework. Executive Summary. COSO. 
Conforti, R., Fortino, G., Rosa, M. L., \& ter Hofstede, A. H. M. (2011). History-Aware, Real-Time Risk Detection in Business Processes. On the Move to Meaningful Internet Systems: OTM 2011, $100-118$.

Cope, E.W., Ku“ ster, J. M., Etzweiler, D., Deleris, L., \& Ray, B. (2010). Incorporating risk into business process models, IBM Journal of Research and Development 54.

Dickstein, D. I., \& Flast, R. H. (2008). No Excuses: A Business Process Approach to Managing Operational Risk, Wiley, Hoboken, N.J, 1 edition.

Dumas, M., La Rosa, M., Mendling, J., \& Reijers, H. A. (2018). Fundamentals of Business Process Management, Springer Berlin Heidelberg, Berlin, Heidelberg.

Ferreira, F. S. (2016). R-BPM: uma metodologia para gestão de riscos em iniciativas de BPM. Dissertação de mestrado, Centro de Informática, Universidade Federal de Pernambuco, Recife, PE, Brasil.

Ferreira, F. S., Alves, C. F., \& Cavalcanti, R. C. (2016). R-BPM: Uma Metodologia para Gerenciamento de Processos de Negócios Consciente dos Riscos. Revista Brasileira de Sistemas de Informação, Rio de Janeiro, vol. 9, No. 4, pp. 05-37.

Gong, Y., \& Janssen, M. (2012). From prolicy implementation to business process management: Principles for creating flexibility and agility. Government Information Quaterly, 29, 61-71.

Haggag, M. H., Khedr, A. E., \& Montasser, H. S. (2015). A risk-aware business process management reference model and its application in an egyptian university. International Journal of Computer Science \& Engineering Survey (IJCSES), 6(2), 11-27.

Hall, D. (2001). Balancing Project Risks and Opportunities. In Proceedings of the Project Management Institute Annual Seminars \& Symposium, Nashville, USA.

Hammer, M. (2010). What is Business Process Management? In Brocke, J., \& Rosemann, M. Handbook on Business Process Management 1: Introduction, Methods, and Information Systems (pp. 316). Heidelberg: Springer.

Hofstede, A. (2011). Risk-Aware Business Process Management.

Instituto Brasileiro de Governança Corporativa. IBGC. (2017). Guia de Orientação para Gerenciamento de Riscos Corporativos. São Paulo: Instituto Brasileiro de Governança Corporativa.

Kim, J., Lee, J., \& Choi, I. (2017). An integrated process-related risk management approach to proactive threat and opportunity handling: A framework and rule language, Knowledge and Process Management 24, 23-37.

Lamine, E., Thabet, R., Sienou, A., Bork, D., Fontanili, F., \& Pingaud, H. (2020). BPRIM: An integrated framework for business process management and risk management, Universität Wien Volume 117.

Mahmood, K., Kangilaski, T., \& Shevtshenko, E. (2018). Usage of Process Models for Quality Management System: A Case Study of Energy Company Department of Mechanical and Industrial Engineering, Tallinn University of Technology, Estonia. 
Oliveira, L. (2014). EBPM: uma metodologia para gestão de processos de negócio. Dissertação de mestrado, Centro de Informática, Universidade Federal de Pernambuco, Recife, PE, Brasil.

Rosemann, M., \& Muehlen, M. Z. (2005). Integrating Risks in Business Process Models, ACIS 2005 Proceedings.

Sienou, A., Lamine, F., Pingaud, H., \& Karduck, A. (2010, Abril). Risk driven process engineering in digital ecosystems: Modelling risk. Digital Ecosystems and Technologies. Digital Ecosystems and Technologies (DEST), 2010 4th IEEE International Conference on, 647 - 650.

Suriadi, S., Weiß, B., Winkelmann, A., Hofstede, A. H. M., \& Adams, M. (2014). Current Research in Risk-aware Business Process Management Overview, Comparison, and Gap Analysis. Communications of the Association for Information Systems, CAIS, 34(52), 2-55.

Shah, L. A., Etienne, A., Siadat, A., \& Vernadat, F. (2017) Process-oriented risk assessment methodology for manufacturing process evaluation, International Journal of Production Research 55.

Valeriano, Dalton L. Gerência em projetos: pesquisa, desenvolvimento e engenharia. São Paulo: Makron Books, 1998.

Varela-Vaca, A. J. (2016). Opbus: A framework for improving the dependability of risk-aware business processes, Al Communications 29. 